NOTE ICHTYOLOGIQUE

\title{
ANGUILLICOLA, UNE ÉPIZOOTIE PLURISPÉCIFIQUE EN EUROPE APPARITION DE ANGUILLICOLA CRASSA \\ (NEMATODA, ANGUILLICOLIDAE) CHEZ L'ANGUILLE EUROPÉENNE ANGUILLA ANGUILLA EN CAMARGUE, SUD DE LA FRANCE
}

\author{
Francis DUPONT (1) Annie J. PETTER (2)
}

(1) Laboratoire de Parasitologie Comparée (U.A. CNRS 698) Université des Sciences et Techniques du Languedoc, PI. E. Bataillon, 34060 MONTPELLIER Cedex, France.

(2) Laboratoire des vers, Muséum National d'Histoire Naturelle, 61, rue Buffon, 75231 PARIS cedex 05. France.

Reçu le 9 juillet 1987

Accepté le 24 mars 1988

Received 9 July, 1987

Accepted 24 March, 1988

\section{RÉSUMÉ}

Nous signalons pour la première fois en France la présence d'une espèce de Nématode du genre Anguillicola, $A$. crassa, rencontrée chez deux populations naturelles d'anguilles, en Camargue (Sud de la France); des compléments sont apportés à la description originale de l'espèce. Une prévalence de $96 \%$ a été notée à l'étang du Grand Palun. La question de la propagation et de la pathogénicité de ce Nématode, parasite de la vessie gazeuse, est abordée.

\section{ANGUILLICOLA, A MULTISPECIFIC EPIZOOTIC IN EUROPE APPEARANCE OF ANGUILLICOLA CRASSA (NEMATODA, ANGUILLICOLIDAE) IN THE EUROPEAN EEL ANGUILLA ANGUILLA IN CAMARGUE, SOUTHERN FRANCE}

\section{SUMMARY}

A species of the Nematode genus Anguillicola, A. crassa is reported for the first time in France in two natural populations of eels in Camargue (Southern France); some data are added to the original description of the species. A prevalence of $96 \%$ has been noted in the lagoon of Grand Palun. The question of the propagation and the pathogenicity of this parasitic Nematode of the swimbladder is discussed.

\section{INTRODUCTION}

Pour la première fois en Europe, en 1982, Paggi et al., rapportent la présence d'un Nématode du genre Anguillicola, A. australiensis chez l'anguille européenne Anguilla anguilla, au lac de Bracciano (Italie). Quelques années plus tard, Anguillicola sp. est signalé chez les anguilles de plusieurs rivières d'Allemagne (NEUMAN, 1985; PETERS et HARTMAN, 1986,) et $A$. crassa en Belgique (BELPAIRE et al., 1987) et en Italie (CANESTRI-TROTTI, 1987). Nous signalons cette fois la présence d'Anguillicola crassa dans les populations naturelles d'anguilles de Camargue (Delta du Rhône, Sud de la France).

Le genre Anguillicola comprend 3 espèces : 2 espèces asiatiques (Chine, Japon), A. globiceps (YAMAGUTI, 1935) parasite de Anguilla japonica et $A$. crassa (KUWAHARA, NIIMI et ITAGAKI, 1974) trouvée dans les élevages d'anguilles japonaises et européennes, au Japon, et 1 espèce australienne A. australiensis (JOHNSTON et MAWSON, 1940), décrite chez Anguilla reinhardtii. Quelque soit l'espèce, ces Nématodes vivent dans la vessie gazeuse des hôtes infestés, se nourissant de sang. Leur cycle biologique, étudié chez A. globiceps et $A$. crassa, comporte un copépode pour hôte intermédiaire (WANG PUKIN et ZHAO YURU, 1980; HIROSE et al., 1976). 


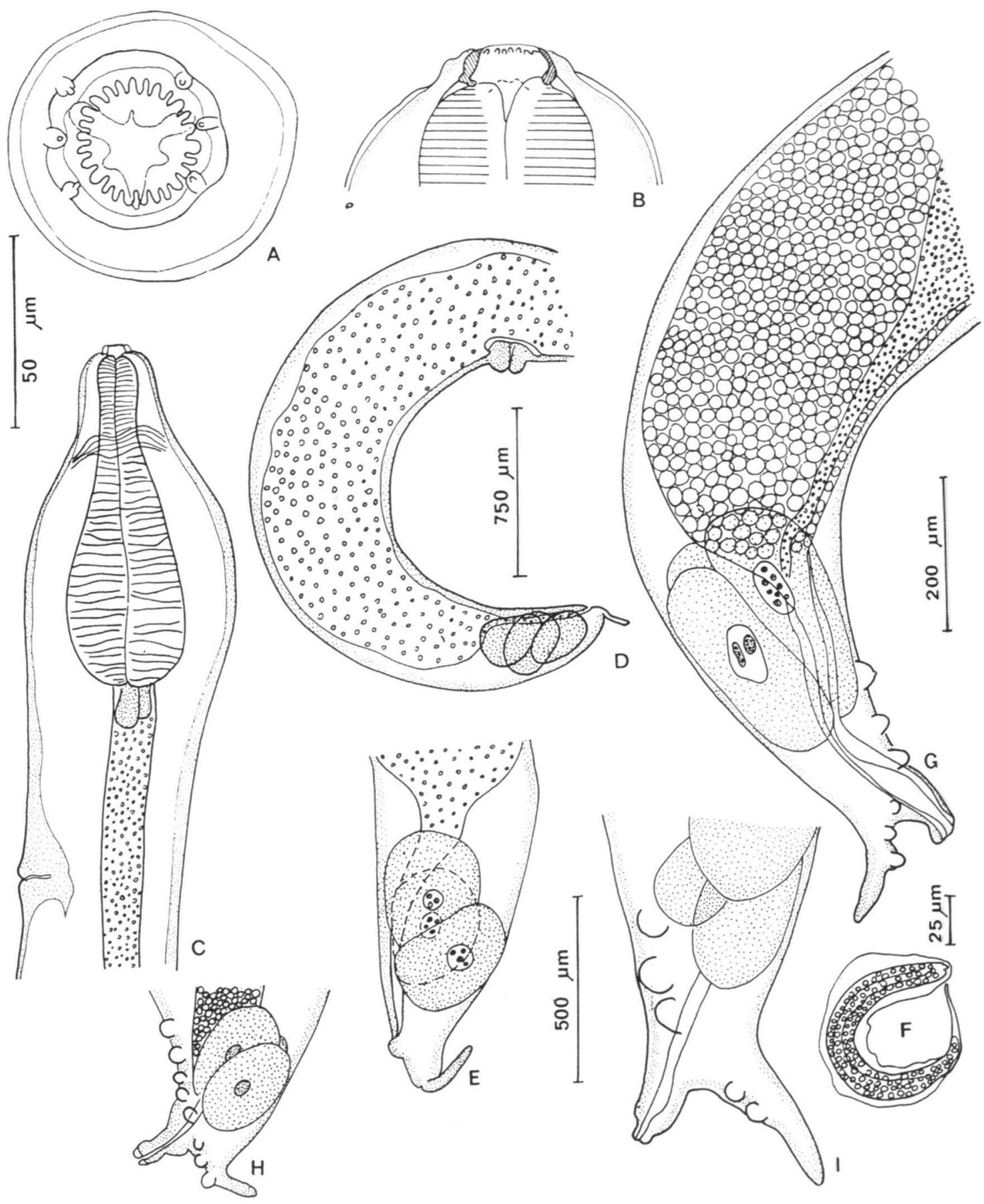

Figure 1 : Anguillicola crassa

A : vue apicale; $B$ : extr. ant., vue dorsale; $C$ : région ant., vue latérale; $D$ : femelle, région post., vue latérale; $\mathbf{E}$ : femelle, extr. post., vue latérale; F : larve; G. H, I: mâles, ext. post., vues latérales.

Figure 1 : Anguillicola crassa

A ; apical view ; B : anterior extremity, dorsal view ; C : anterior part, lateral view ;

D : female, posterior part, lateral view ; E : female, posterior extremity, lateral view;

F : larva ; G, H, I : males, posterior extremities, lateral views.

\section{ÉCHELLE}

A : éch. $50 \mu \mathrm{m}$; B. F : éch. $25 \mu \mathrm{m}$; C, E, H: éch. $500 \mu \mathrm{m}$; D: éch. $750 \mu \mathrm{m}$; G, I: éch. $200 \mu \mathrm{m}$. 


\section{DESCRIPTION DE ANGUILLICOLA CRASSA}

Les Nématodes que nous avons découverts en Camargue appartiennent tous à l'espèce A. crassa. Les mâles mesurent de $13 \mathrm{~mm}$ à $28 \mathrm{~mm}$ et les femelles de $20 \mathrm{~mm}$ à $55 \mathrm{~mm}$ (10 spécimens mesurés pour chaque sexe) ; les dimensions des différents organes correspondent à celles qui sont indiquées dans la description originale, la seule différence avec celle-ci réside dans le nombre des papilles caudales du mâle: chez nos spécimens, ce nombre est généralement de 6 paires (fig. 1, G), alors que la description originale (KUWAHARA et al, 1974) en signale 5 paires ; cependant, chez 2 spécimens, le côté gauche présente respectivement 8 papilles et 5 papilles (fig. 1, H et I), ce caractère ne nous semble donc pas suffisant pour séparer nos spécimens de Anguillicola crássa.

Nous donnons une planche de figures et une description de l'extrémité apicale qui n'est pas étudiée dans la description originale.

La bouche est circulaire, il existe 2 amphides et 4 papilles submédianes; la capsule buccale est munie antérieurement de 26 petites dents; au fond de la capsule buccale, s'observent 3 lobes œsophagiens bilobés (fig. 1, A)

L'espèce A. crassa se différencie de A. australiensis, l'autre espèce d'Anguillicola signalée en Europe, par l'absence de dilatation en forme du bulbe à l'extrémité antérieure.

\section{DONNÉES ÉPIDÉMIOLOGIQUES}

En automne 1985, l'examen de 142 anguilles (en majoritè des mâles argentés mesurant en moyenne $370 \mathrm{~mm}$ de long) capturées dans l'Étang du Grand Palun (Camargue) a mis en évidence l'importance épidémiologique de $A$. crassa dans la population d'anguilles: le parasite est présent chez $96 \%$ des anguilles et occupe généralement la totalité du volume disponible (jusqu'à 30 individus par vessie gazeuse, provoquant un épaississement de la paroi). A la même époque, une prévalence de $32 \%$ est notée sur un échantillon de 25 anguilles prélevées dans le canal de Fumemorte (Camargue). En 1982-83, la population d'anguilles du canal fut l'objet d'une étude parasitologique (DUPONT, 1984). La présence de ce Nématode n'a jamais été notée. II est donc probable que l'apparition de $A$. crassa, aú canal du Fumemorte, est postérieure à l'année 1983.

\section{DISCUSSION ET CONCLUSION}

De façon générale, il paraît peu vraisemblable que la découverte récente du genre Anguillicola en Europe soit fortuite, c'est-à-dire due au manque d'investigations parasitologiques dans le passé. Au contraire, l'apparition soudaine puis l'extension rapide du genre Anguillicola en Europe sont les marques d'une épizootie de nature multispécifique puisqu'interviennent 2 ou 3 espèces: A. australiensis en Italie, Anguillicola sp. en Allemagne, A. crassa en France, en Belgique et en Italie.

Ce parasite semble se développer uniquement en milieux dulçaquicole et oligohalin (cas de l'Étang du Grand Palun et du canal du Fumemorte). Les copépodes pélagiques de ces milieux jouent vraisemblablement un rôle fondamental dans la transmission parasitaire. HIROSE et al., (1976) ont montré notamment que le Copépode Eucyclops serrulatus constituait expérimentalement un hôte intermédiaire possible de $A$. crassa. Or cette espèce est très cosmopolite en Europe. Le fait que les copépodes constituent un élément négligeable de la nourriture de l'anguille n'exclut pas - comme l'ont suggéré PETERS et HARTMAN (1986) - que ceux-ci jouent un rôle essentiel dans les cycles parasitaires de l'anguille. En effet, DUPONT et GABRION (1986) ont montré chez un Cestode Pseudophyllide de l'anguille européenne, le rôle capital des copépodes, hôtes intermédiaires, et celui, plus secondaire (non indispensable) des poissons, hôtes paraténiques, dans la circulation du parasite.

En accord avec PAGGl et al., (1982) et PETERS et HARTMAN (1986), on peut supposer que l'introduction d'Anguillicola en Europe soit la conséquence d'importations d'anguilles d'origine asiatique. En Camargue, la découverte sur l'anguille d'un Monogène décrit au Japon Pseudodactylogyrus anguillae (LAMBERT et al., 1985) renforce cette opinion. Cependant, en dépit d'une enquête menée autour de la Camargue, l'introduction d'anguilles d'origine japonaise n'a pu être décelée (XIMENES, com. pers.).

La pathogénicité des ces Nématodes est encore inconnue. On peut néanmoins envisager qu'ils exercent deux types d'actions néfastes, spoliatrice en raison de leur régime hématophage et perturbatrice de la fonction respiratoire, toujours hypothétique, accordée à la vessie gazeuse (cf. TESCH, 1977). PETERS et HARTMAN (1986) ajoutent que ces Nématodes réduisent la résistance des anguilles en captivité et posent des problèmes de transport et de vente.

Le recrutement des anguilles destinées à l'élevage est totalement dépendant de l'existence de populations naturelles. Ce fait impose de connaître les traits épidémiologiques de l'infection des populations sauvages d'anguilles si l'on désire éviter le développement de ces Nématodes dans les élevages. II serait notamment intéressant de déterminer les facteurs du milieu limitant l'accomplissement du cycle parasitaire (comme la salinité) 


\section{RÉFÉRENCES}

BELPAIRE C., DE CHARLEROY D., THOMAS K., (1987). - Effects of restocking eels on the distribution of the Nematode Anguillicola crassa in Flanders, Belgium. EIFAC Working Party on Eel, Bristol, United Kingdom, 13-16 Avril.

CANESTRI-TROTTI G., (1987). - Occurence of the Nematode Anguillicola crassa (Kuwahara, Niimi \& Itagaki, 1974) in eels from the Po delta, Italy. EIFAC Working Party on Eel, Bristol, United Kingdom, 13-16 Avril.

DUPONT F., (1984). - Biologie des populations de Bothriocephalus claviceps Cestode Pseudophyllidea parasite de l'anguille européenne. Thèse $3^{\circ}$ cycle, U.S.T.L., Montpellier: $199 \mathrm{pp}$.

DUPONT F. et GABRION C.,(1986) - Approche expérimentale du rôle de l'hôte paraténique dans la circulation du parasite Bothriocephalus claviceps (Cestode, Pseudophyllidea). Ann. Parasitol. Hum. Comp., 61 : 423-429.

HIROSE H., SEKINO T., EGUSA S., (1976). - Notes on the egg deposition, larval migration and intermediate host of the Nematode Anguillicola crassa parasitic in the swimbladder of eels. Fish Pathol., 11: 27-31.

KUWAHARA A., NIIMI A., ITAGAKI H., (1974). - Studies on a Nematode parasitic in the air bladder of the eel. I - Description of Anguillicola crassa n. sp. (Philometridea, Anguillicolidae). Jap. J. Parasit., $23: 275-279$.

LAMBERT A., LE BRUN N., PARISELLE A., (1985). - Présence en France de Pseudodactylogyrus anguillae (Yin et Sproston, 1948) Gussev, 1965 (Monogenea, Monopisthocotylea), parasite branchial de l'anguille européenne Anguilla anguilla en eau douce. Ann. Parasitol. Hum. Comp., $60: 91-92$.

NEUMAN W., (1985). - Schwimmblasenparasit Anguillicola bei Aalen. Fischer und Teichwirt, $11: 322$.

PAGGI L., ORECCHIA P., MINERVINI R., MATIUCCI S., (1982). - Sulla compensa di Anguillicola australiensis Johnston et Mawson, 1940 in Anguilla anguilla del lago di Bracciano. Parasitologia, 24 : 139-144.

PETERS G., HARTMAN F., (1986). - Anguillicola, a parasitic Nematode of the swimbladder spreading among eel populations in Europe. Dis. aquat. Org., $1: 229-230$.

TESCH F. - W. (1977). - The eel biology and management of Anguillid eels. Chapman and Hall, Ltd: $434 \mathrm{pp}$.

WANG PUKIN, ZHAO YURU, (1980). - Observations on the life-history of Anguillicola globiceps (Nematoda: Anguillicolidae). Acta Zoologica Sinica, $26: 243-249$. 